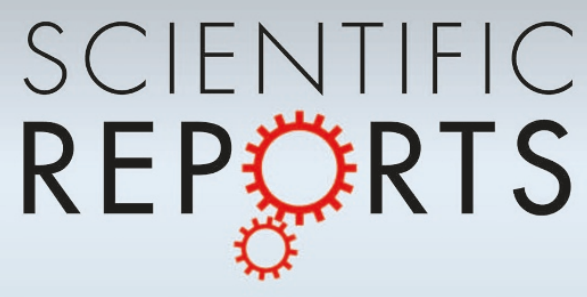

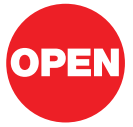

SUBJECT AREAS:

STATISTICAL PHYSICS, THERMODYNAMICS AND NONLINEAR DYNAMICS

THEORETICAL PHYSICS

THEORY AND COMPUTATION

PHYSICS

Received

10 July 2012

Accepted

14 September 2012

Published

4 October 2012

Correspondence and requests for materials should be addressed to L.H. (huangl@|zu.edu.

$\mathrm{cn})$

\section{Emergence of grouping in multi-resource minority game dynamics}

\author{
Zi-Gang Huang', Ji-Qiang Zhang', Jia-Qi Dong', Liang Huang ${ }^{1,2} \&$ Ying-Cheng Lai ${ }^{2,3}$
} ${ }^{1}$ Institute of Computational Physics and Complex Systems, Lanzhou University, Lanzhou, Gansu 730000, China, ${ }^{2}$ School of
Electrical, Computer, and Energy Engineering, Arizona State University, Tempe, AZ 85287, USA, ${ }^{3}$ Department of Physics, Arizona
State University, Tempe, AZ 85287, USA.

Complex systems arising in a modern society typically have many resources and strategies available for their dynamical evolutions. To explore quantitatively the behaviors of such systems, we propose a class of models to investigate Minority Game (MG) dynamics with multiple strategies. In particular, agents tend to choose the least used strategies based on available local information. A striking finding is the emergence of grouping states defined in terms of distinct strategies. We develop an analytic theory based on the mean-field framework to understand the "bifurcations" of the grouping states. The grouping phenomenon has also been identified in the Shanghai Stock-Market system, and we discuss its prevalence in other real-world systems. Our work demonstrates that complex systems obeying the MG rules can spontaneously self-organize themselves into certain divided states, and our model represents a basic and general mathematical framework to address this kind of phenomena in social, economical and political systems.

the he Minority Game (MG) was originated from the El Farol bar problem in game theory first conceived by Arthur in $1994^{1}$, where a finite population of people try to decide, at the same time, whether to go to the bar on a particular night. Since the capacity of the bar is limited, it can only accommodate a small fraction of all who are interested. If many people choose to go to the bar, it will be crowded, depriving the people of the fun and thereby defying the purpose of going to the bar. In this case, those who choose to stay home are the winners. However, if many people decide to stay at home then the bar will be empty, so those who choose to go to the bar will have fun and they are the winners. Apparently, no matter what method each person uses to make a decision, the option taken by majority of people is guaranteed to fail and the winners are those that choose the minority strategy. Indeed, it can be proved that, for the El Farol bar problem there are mixed strategies and a Nash-equilibrium solution does exist, in which the option taken by minority wins ${ }^{2}$. A variant of the problem was subsequently proposed by Challet and Zhang, named as a MG problem ${ }^{3}$, where a player among an odd number of players chooses one of the two options at each time step. Subsequently, the model was studied in a series of works ${ }^{4-22}$. In physics, MG has received a great deal of attention from the statistical-mechanics community, especially in terms of problems associated with non-equilibrium phase transitions ${ }^{23-25}$.

In the current literature, the setting of MG is that there is a single resource but players have two possible strategies (e.g., in the El Farol bar problem there is a single bar and the two strategies are going to the bar or staying at home), and an agent is assumed to react to available global information about the history of the system by taking on an alternative strategy that is different than its current one. An outstanding question remains of the nonlinear dynamics of MG with multiple resources. The purpose of this paper is to present a class of multi-resource MG models. In particular, we assume that, at any time, an individual agent has $k>1$ resources/strategies to choose from. We introduce a parameter $p$, which is the probability that each agent reacts based on the available local information by selecting a less crowded resource in an attempt to gain higher payoff. Here we assume realistically that only local information about the immediately preceding step is available, which constitutes the input to the model. This differs from the original MG model where global information is assumed to be available to all the agents and they make actions based on the past history. We call $p$ the minority-preference probability. We find that, as $p$ is increased, the striking phenomenon of grouping emerges, where the resources can be distinctly divided into two groups according to the number of their attendees. In addition, the number of stable pairs of groups also increases. We will demonstrate the phenomenon numerically and derive an analytic theory to fully explain the phenomenon. We will also show 
that the grouping phenomenon plays a fundamental role in shaping the fluctuations of the system. An application to a real financialmarket system by analyzing the available empirical data will also be illustrated, where grouping of stocks (resources) emerges. Our model is not only directly relevant to nonlinear and complex dynamical systems, but also applicable to social and economical systems.

Our multi-resource MG model and the emergence of grouping phenomenon are presented in the Results section. A general theory is also developed in the Results section to elucidate the dynamics of the emergence and evolution of the strategy groups, together with a discussion of relevance to real-world systems. Concluding remarks are presented in the Discussion section.

\section{Results}

Multi-resource minority game model. We consider a complex, evolutionary-game type of dynamical system of $N$ interacting agents competing for multiple resources. Each agent chooses one resource in each round of the game. Each resource has a limited capacity, i.e., the number of agents it can accommodate has an upper bound $n_{c}$. There are thus multiple strategies $(s=1,2, \ldots, k$, where $k$ is the maximum number of resources/strategies) available to each agent. On average, each strategy can accommodate $N / k$ agents, and we consider the simple case of $n_{c}=N / k$. Let $n_{s}$ be the number of agents selecting a particular strategy $s$. If $n_{s} \leq n_{c}$, the corresponding agents win the game and, consequently, $s$ is the minority strategy. However, if $n_{s}>n_{c}$, the associated resource is too crowded so that the strategy fails and the agents taking it lose the game, which defines the "majority strategy." The optimal solution of the game dynamics is thus $n_{s}=n_{c}$.

In a real-world system, it is often difficult or practically impossible for each agent to gain global information about the dynamical state of the whole system. It is therefore useful to introduce the concept of local information network in our multipleresource MG model. At each time step, with probability $p$, namely the minority-preference probability, each agent acts based on local information that it gains by selecting one of the $k$ available strategies. In contrast, with probability $1-p$, an agent acts without guidance of any local information. For the minority-preference case, agent $i$ has $d$ neighbors in the networked system. The required information for $i$ to react consists of all its neighbors' strategies and, among them, the winners of the game, i.e., those neighboring agents choosing the minority strategies at the last time step. Let $\Pi=\left\{s_{m}\right\}$ be the set of minority strategies for $i$ s winning neighbors, where a strategy may appear a number of times if it has been chosen by different winning neighbors. With probability $p$, agent $i$ will chose one strategy randomly from $\Pi$. Thus, the probability $P_{s}$ for strategy $s$ to be selected is proportional to the times it appears in $\Pi$, i.e., $P_{s}=N_{s} / \operatorname{Card}(\Pi)$, where $\operatorname{Card}(\Pi)$ is the number of elements in $\Pi$ and $N_{s}$ the times strategy $s$ appears in $\Pi$. If $\Pi$ is empty, $i$ will randomly select one from the $k$ available strategies. For the case where an agent selects a strategy without any local information with probability $1-p$, it will either choose a different strategy randomly from the $k$ available ones with mutation probability $m^{10}$, or inherit its strategy from the last time step with probability $1-m$.

Numerical results. As a concrete example to illustrate the strategygrouping phenomenon, we set $k=5$. Figures $1(\mathrm{a}-\mathrm{c})$ show time series of $n_{s}$, the number of agents selecting each strategy $s$, for $p=0,0.45$, and 1.0, respectively. For Fig. 1(a) where $p=0$, an agent makes no informed decision in that it changes strategy randomly with probability $m=1$. In this case, $n_{s}$ 's appear random. For the opposite extreme case of $p=1$ [Fig. 1(c)], each agent makes well informed decisions based on available local information about the strategies used by its neighbors. In this case, the time series are quasiperiodic (a detailed analysis will be provided in the Discussion section). For the intermediate case of $p=0.45$ [Fig. 1(b)], agents' decisions are partially informed. In this case, an examination of the time series points to the occurrence of an interesting grouping behavior: the 5 strategies, in terms of their selection by the agents, are divided into two distinct groups $g_{1}$ and $g_{2}$ that contain $k_{g_{1}}=2$ and $k_{g_{2}}=3$ strategies, respectively. The time series associated with the smaller group exhibit larger fluctuations about its equilibrium.

To better characterize the fluctuating behaviors in the time series $n_{s}$, we calculate the variance $\sigma^{2}=\left\langle\left[n_{s}(t)-N / k\right]^{2}\right\rangle$ as a function of the system parameter $p$, where $\langle\cdot\rangle$ is the expectation values averaged over a long time interval, as shown in Fig. 2 on a logarithmic scale. We observe a generally increasing behavior in $\sigma^{2}$ with $p$ and, strikingly, a bifurcation-like phenomenon. In particular, for $p<p_{b}$, where $p_{b}$ is the bifurcation point, $\sigma^{2}$ for all strategies assume approximately the same value. However, for $p>p_{b}$, there are two distinct values for $\sigma^{2}$, signifying the aforementioned grouping behavior [Fig. 1(b)]. From Fig. 2, we also see that, after the bifurcation, the two branches of $\sigma^{2}$ are linear (on a logarithmic scale) and have approximately the same slope $a$, suggesting the following power-law relation: $\sigma_{g_{i}}^{2}=b_{g_{i}} p^{a}$, for $i=1,2$, where $\log \left(b_{g_{1}}\right)$ and $\log \left(b_{g_{2}}\right)$ are the intercepts of the two lines in Fig. 2. We thus obtain

$$
\frac{\sigma_{g_{1}}^{2}}{\sigma_{g_{2}}^{2}}=\frac{b_{g_{1}}}{b_{g_{2}}} .
$$

In the Discussion section, we will develop a theory to explain the relations among the variances of the grouped strategies and to provide formulas for the amplitudes of the time series in Fig. 1 and the sizes (a)

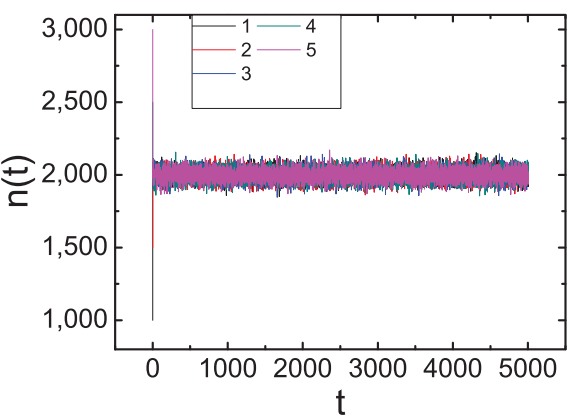

(b)

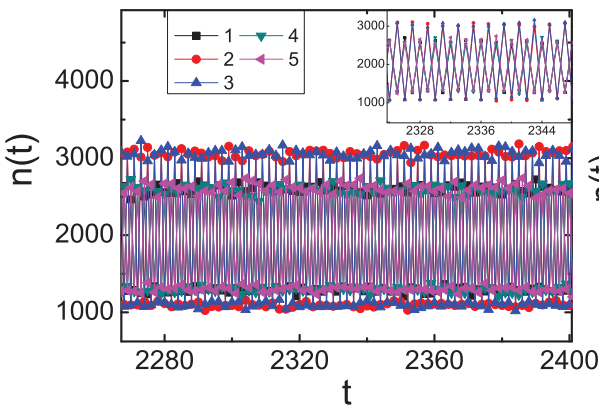

(c)

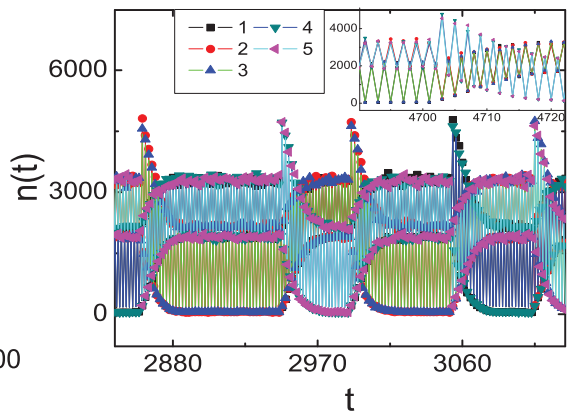

Figure $1 \mid(\mathrm{a}-\mathrm{c})$ For a square-lattice system of $N=10000$ agents, time series of the number of agents selecting each of the $k=5$ available strategies for $p$ $=0,0.45$, and 1.0, respectively. The probability of random alteration of strategy for an agent in the absence of local information

(with probability $1-p$ ) is set to be $m=1$. A strategy grouping behavior can be seen in (b), where the whole strategy set is broken into two groups: one of two and another of three strategies. 


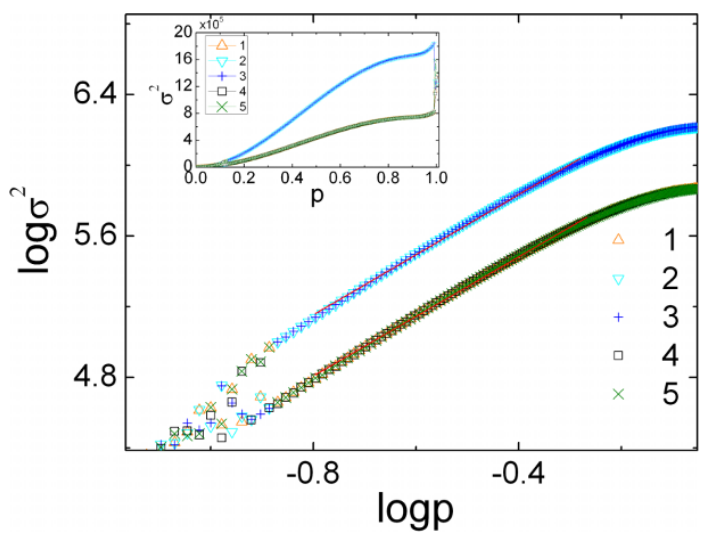

Figure $2 \mid$ For the same system in Fig. 1 , variance $\sigma^{2}$ of the time series $n_{s}$ as a function of $p$ on a logarithmic scale. Different symbols indicate different resources/strategies. A period-doubling like bifurcation occurs, at which a grouping behavior emerges. Inset is the same plot but on a linear scale.

of the groups (denoted by $k_{g_{1}}$ and $k_{g_{2}}$, respectively). Specifically, our theory predicts the following ratio between the variances of the two bifurcated branches:

$$
\frac{\sigma_{g_{1}}^{2}}{\sigma_{g_{2}}^{2}}=\left(\frac{k_{g_{2}}}{k_{g_{1}}}\right)^{2}
$$

which is identical to the numerically observed ratio in Eq. (1), with the additional prediction that the strategies in the group of smaller size exhibit stronger fluctuations since the corresponding value of $\sigma^{2}$ is larger. Overall, the emergence of the grouping behavior in multipleresource MGs, as exemplified in Fig. 2, resembles a period-doubling like bifurcation. While perioddoubling bifurcations are extremely common in nonlinear dynamical systems, to our knowledge, in complex game systems a clear signature of such a bifurcation had not been reported previously.

A careful examination of the time-series for various $p$ has revealed that the strategy-grouping processes has already taken place prior to the bifurcation point $p_{b}$ in the variance $\sigma^{2}$, but all resulted grouping states are unstable. Take as an example the 5-strategy system in Fig. 1. In principle, there can be two types of pairing groups: $(1,4)$ and $(2,3)$. For any grouping state, the following constraint applies:

$$
k_{g_{1}}+k_{g_{2}}=k, \quad k_{g_{1}} \cdot k_{g_{2}} \neq 0
$$

There are in total $k / 2$ (if $k$ is even) or $(k-1) / 2$ (if $k$ is odd) possible grouping states for the system with $k$ available strategies. However, the grouping states are not stable for $p<p_{b}$. What happens is that a strategy can remain in one group but only for a finite amount of time before switching to a different group. Assume that the sizes of the original two pairing groups are $k_{g_{1}}$ and $k_{g_{2}}$, respectively. The sizes of the new pair of groups are thus $k_{g_{1}} \pm 1$ and $k_{g_{2}} \mp 1$, as stipulated by Eq. (3). Associated with switching to a different pair of groups, the amplitudes of the time series $n_{s}$ for each strategy also change. As the bifurcation parameter $p$ is increased, the stabilities of different pairs of grouping states change. At the bifurcation point $p_{b}$, one particular pair of groups becomes stable, such as the grouping state $(2,3)$ in Fig. 2.

The bifurcation-like phenomenon and the emergence of various strategy-grouping states are general for multiple-resource MG game dynamics. For example, Fig. 3 shows $\sigma^{2}$ as a function of $p$ for a system with $k=16$ available strategies. There are in total 8 possible grouping states, ranging from $(8,8)$ to $(1,15)$. As $p$ is increased, the grouping states $(8,8),(7,9),(6,10),(5,11)$ and $(4,12)$ become stable one after another, as can be seen from the appearance of their corresponding branches in Fig. 3. The behavior can be understood theoretically through a stability analysis (the Discussion section).

Another phenomenon revealed by Fig. 3 is the merging of bifurcated branches. For example, as $p$ is increased through about 0.8 , the grouping states disappear one after another in the reverse order as they initially appeared. This can also be understood through the stability analysis (the Discussion section).

A real-world example: emergence of grouping states in financial market. The financial market is a representative multiresource complex system, in which many stocks are available for investment. We analyze the fluctuation of the stock price from the empirical data of 27 stocks in the Shanghai Stock Market's Steel Plate between 2007 and 2010. We regard the 27 stocks, which are mostly from the iron and steel industry, as constituting a MG system with $k=27$ resources, where the agents selecting the resources correspond to the capitals invested. This system is open in the sense that capital typically flows in and out, which is the main difference from our closed-system model. In particular, given the time series $x_{i}(t)$ of the daily closing price of stock $i$, the daily log-return is defined as $R_{i}(t)=$ $\ln x_{i}(t)-\ln x_{i}(t-1)$. The average return of the 27 stocks at time $t$, denoted by $\left\langle R_{i}(t)\right\rangle$, signifies a global trend of the system at $t$, which is caused by the change in the total amount of the capital in this open, 27-stock system. However, when we analyze the detrended logreturns $R_{i}^{\prime}(t)=R_{i}(t)-\left\langle R_{i}(t)\right\rangle$, the system resembles a closed system, as in our model. We shall demonstrate that the strategygrouping phenomenon occurs in this real-world system.

We calculate the Pearson parameter $c_{i j}$ of each pair of the detrended log-returns $R_{i}^{\prime}(t)$ and $R^{\prime}{ }_{j}(t)$, which leads to a $k \times k$ correlation matrix $C$, as shown in Fig. 4(a). In terms of the eigenvector associated with the maximum eigenvalue of matrix $C$, we rank the (a)

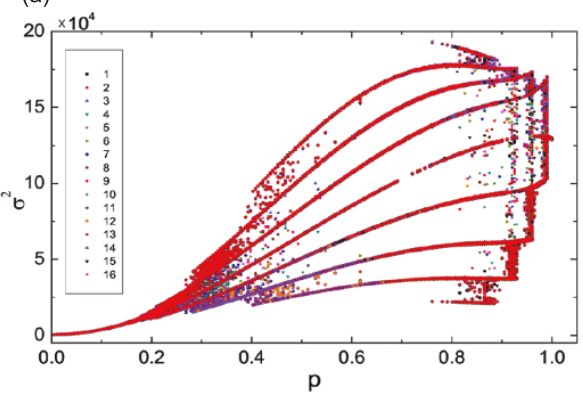

(b) $\times 10^{5}$

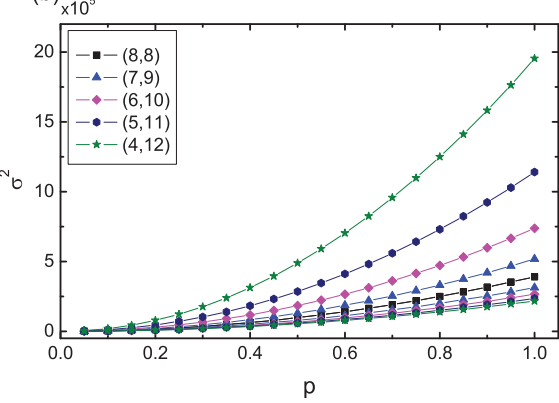

(c) $\times 10^{4}$

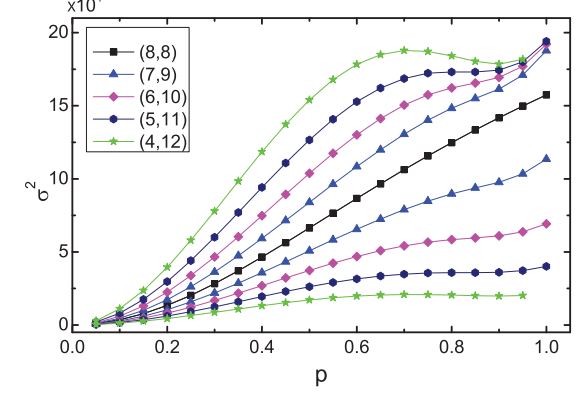

Figure $3 \mid$ For a multiple-resource MG system of $N=10000$ agents on a square lattice and $k=16$ available strategies, (a) numerically obtained bifurcation-like behavior that leads to the emergence of various pairs of grouping states $[\mathrm{e.g},(8,8),(4,12)$, etc.], (b) predicted bifurcation from mean-field theory (the Discussion section), and (c) results from an improved mean-field theory (the Discussion section). The probability of random selection in the absence of local information is set to be $m=1$. 
(a)
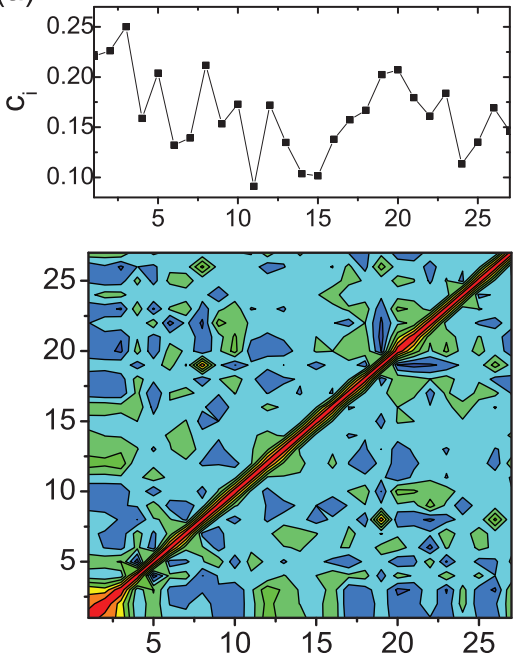

(b)
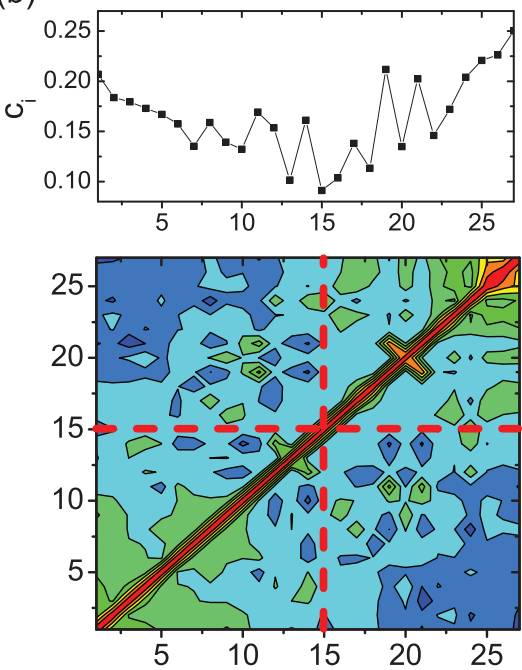

(c)
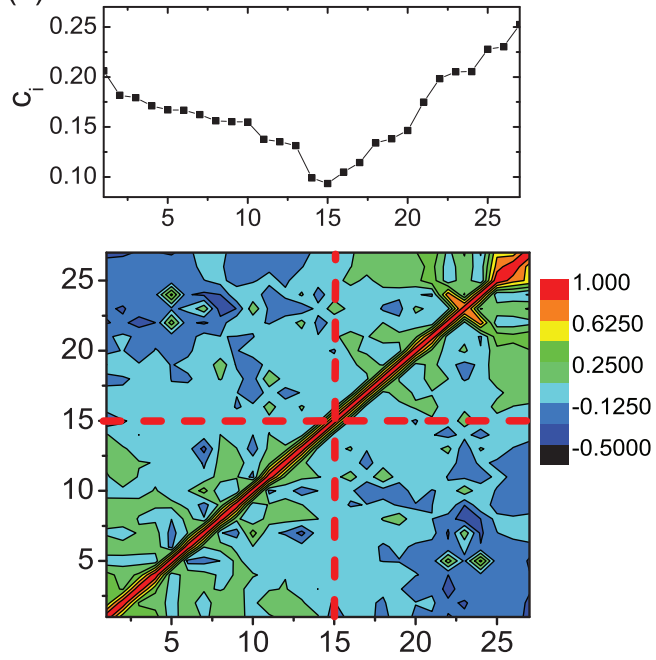

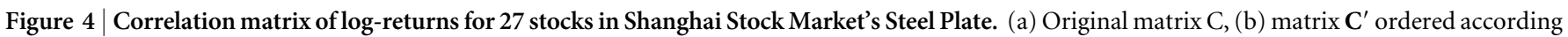

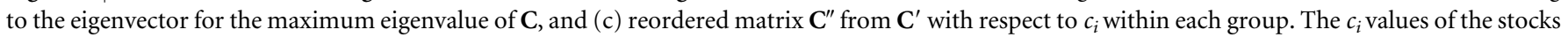
are shown for each matrix.

order of the stocks and obtain matrix $\mathrm{C}^{\prime}$, as shown in Fig. 4(b). The striking behavior is that the matrix is apparently divided into 4 blocks, a manifestation of the grouping phenomenon. In particular, the matrix elements $c_{i j}$ among the first 15 stocks and those among the remaining 12 stocks are generally positive, but the cross elements between stocks in the two groups are negative. It is thus quite natural to classify the first 15 stocks as belonging to group $g_{1}$ and the remaining 12 to group $g_{2}$. We can then write the matrix in a block form as

$$
\mathbf{C}^{\prime}=\left(\begin{array}{ll}
\mathbf{C}_{g_{2} g_{1}} & \mathbf{C}_{g_{2} g_{2}} \\
\mathbf{C}_{g_{1} g_{1}} & \mathbf{C}_{g_{1} g_{2}}
\end{array}\right),
$$

where the elements of $\mathbf{C}_{g_{1} g_{1}}$ and $\mathbf{C}_{g_{2} g_{2}}$ are positive, and those of $\mathbf{C}_{g_{1} g_{2}}$ and $\mathbf{C}_{g_{2} g_{1}}$ are negative. The phenomenon is that the 27-stock system has self-organized itself into a $(12,15)$ grouping state, a natural consequence of the MG dynamics in multi-resource complex systems.

For one given stock $i$, the mean absolute correlation is

$$
c_{i}=\sum_{j=1}^{k}\left|c_{i j}\right| /(k-1), \quad j \neq i .
$$

This parameter reflects the weight of the stock in the system. If $c_{i} \rightarrow 0$, oscillations of stock $i$ are contained in the noise floor. In this case, there is no indication as to whether this stock belongs to group $g_{1}$ or $g_{2}$. The larger the value of $c_{i}$, the less ambiguous that the stock belongs to either one of the two groups. From the value of $c_{i}$ ranked in the same order as in $\mathrm{C}^{\prime}$, we can see that the boundary of the two groups is the stock with minimum $c_{i}$. Thus $c_{i}$ can be considered as a characteristic number to distinguish different groups. We can also reorder the matrix $\mathbf{C}^{\prime}$ according to $c_{i}$ within group $g_{1}$ and $g_{2}$, respectively. This leads to matrix $\mathrm{C}^{\prime \prime}$, as shown in Fig. 4(c), further demonstrating the grouping phenomenon.

Generality of strategy-grouping phenomenon in multi-resource MG systems. We have observed numerically from model MG systems with multiple resources the grouping phenomenon in terms of the emergence, characteristics, and evolutions of the strategy groups. An issue of discussion is whether the phenomenon is generic. To address this issue and also to gain a comprehensive understanding of multiple-resource MG systems in general, we develop an analytic theory.
Relationship among variance, amplitude and group size. In general, for a multiple-resource MG system of $N$ agents, as the parameter $p$ is increased so that agents become more likely to make informed decision for strategy selection, the available strategies can be divided into pairs of groups. The example in Fig. 1(b) presents a case where there are two distinct strategy groups $g_{1}$ and $g_{2}$, which contain $k_{g_{1}}$ and $k_{g_{2}}$ strategies, respectively, where $k_{g_{1}}+k_{g_{2}}=k$. For Fig. 1(b), we have $k_{g_{1}}<k_{g_{2}}$. The strategies belonging to the same group are selected by approximately the same number of agents, i.e., the time series $n_{s}(t)$ for strategies in the same group are nearly identical. During the time evolution, a strategy $s$ can switch iteratively from being a minority strategy $\left[n_{s}(t)<n_{c} \equiv N / k\right]$ to being a majority one $\left[n_{s}(t+1)>n_{c}\right]$. In particular, as shown in the schematic map in Fig. 5, for the strategy in group $g_{1}$ denoted by $s_{i}$, and the strategy in group $g_{2}$ denoted by $s_{j}$, if $n_{s_{i}}(t)<N / k<n_{s_{j}}(t)$, we will have $n_{s_{i}}(t+1)>N / k>n_{s_{j}}(t+1)$. In addition, the time series $n_{s}$ reveals that the average numbers of agents for strategies $s_{i}$ and $s_{j}$, denoted by $\left\langle n_{s_{i}}\right\rangle$ and $\left\langle n_{s_{j}}\right\rangle$ (the blue dash line and red dot line in Fig. 5), respectively, are not equal to $N / k$ (the black solid line in Fig. 5). In fact, we have

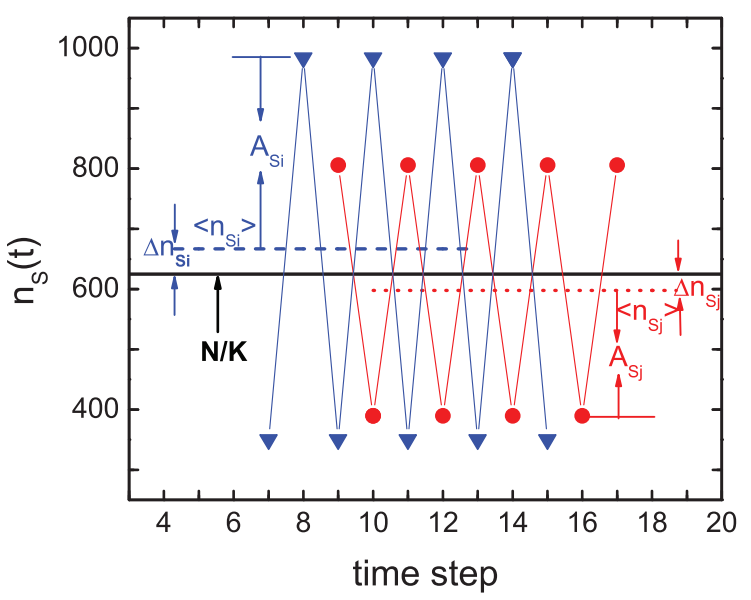

Figure 5 | For the case of two groups [e.g., Fig. 1(b)], schematic illustration of time series $n_{s_{i}}$ in group $g_{1}$ (blue circles) and $n_{s j}$ in group $g_{2}$ (red triangles), where various quantities such as the amplitude $A$ are labelled. 


$$
\left\langle n_{s_{i}}\right\rangle=\frac{N}{k}+\Delta n_{s_{i}}, \quad\left\langle n_{s_{j}}\right\rangle=\frac{N}{k}-\Delta n_{s_{j}}
$$

where $\left\langle n_{s_{i}}\right\rangle>N / k$ and $\left\langle n_{s_{j}}\right\rangle<N / k$. Here, $\Delta n_{s_{x}}$ is the absolute value of the difference between $\left\langle n_{s_{x}}\right\rangle$ and $N / k$. The number of agents $n_{s_{i}}(t)$ [or $n_{s_{j}}(t)$ ] typically fluctuates about the equilibrium $\left\langle n_{s_{i}}\right\rangle$ [or $\left.\left\langle n_{s_{j}}\right\rangle\right]$ with amplitude $A_{s_{i}}$ [or $A_{s_{j}}$, as shown in the schematic map in Fig. 5.

Based on the numerical observations, we can argue that the strategy grouping phenomenon is intimately related to the fluctuations in the time series $n_{s}$. Assuming the MG system is closed so that the number of agents is a constant, we can obtain (see Supplementary Information for detail)

$$
\frac{A_{s_{i}}}{A_{s_{j}}}=\frac{k_{g_{2}}}{k_{g_{1}}}, \quad \frac{\Delta n_{s_{i}}}{\Delta n_{s_{j}}}=\frac{k_{g_{2}}}{k_{g_{1}}}, \quad \frac{\sigma_{s_{i}}^{2}}{\sigma_{s_{j}}^{2}}=\left(\frac{k_{g_{2}}}{k_{g_{1}}}\right)^{2} .
$$

We see that the fluctuations of the time series are closely related to the grouping of the strategies. As shown in Fig. 2, the ratio of the variances of group $g_{1}$ and $g_{2}$ from the simulation agree very well with Eq. (5).

Mean-field theory. In Supplementary Information, we also develop a mean-field theory, as well as its modified version to understand the fluctuation patterns of the system. The mean-field treatment gives the iterative dynamics of agents selecting strategy $s_{i}$ in terms of the values of $n_{s_{i}}$, the expression of the amplitude $A_{s_{i}}$ of the fluctuation, the mean value $\left\langle n_{s_{i}}\right\rangle$, and its difference from $N / k$, as follows:

$$
\begin{aligned}
& n_{s_{i}}^{(2 a)}=\frac{\alpha \beta+\beta+\gamma_{g_{1}}}{1-\alpha^{2}}, \\
& n_{s_{i}}^{(2 a+1)}=\frac{\alpha \beta+\alpha \gamma_{g_{1}}+\beta}{1-\alpha^{2}},
\end{aligned}
$$

and,
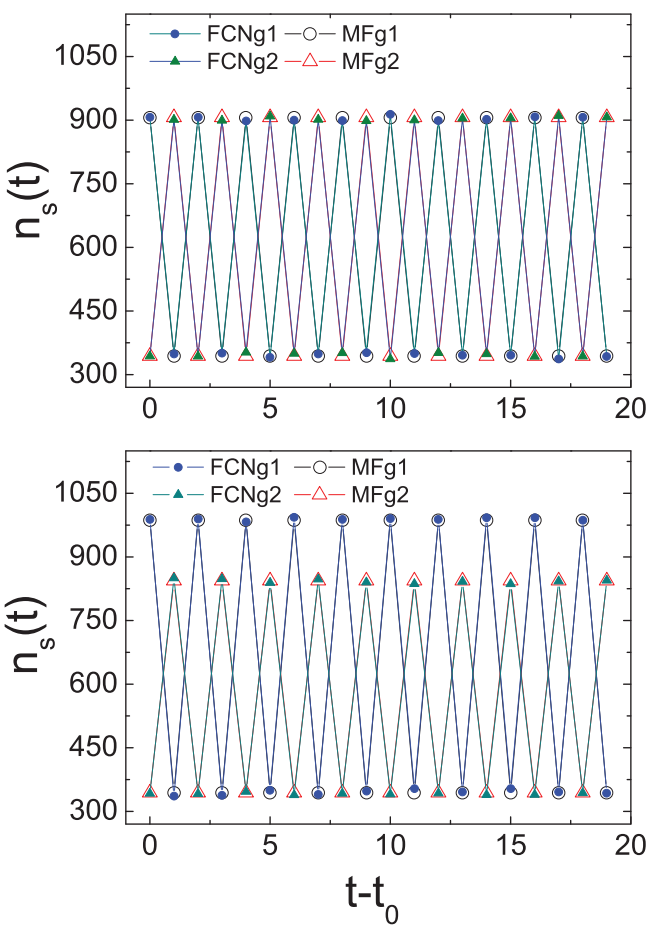

$$
\begin{aligned}
& A_{s_{i}}=\left[n_{s_{i}}^{(2 a)}-n_{s_{i}}^{(2 a+1)}\right] / 2=\frac{\gamma_{g_{1}}}{2(1+\alpha)}, \\
& \left\langle n_{s_{i}}\right\rangle=\left[n_{s_{i}}^{(2 a)}+n_{s_{i}}^{(2 a+1)}\right] / 2=\frac{2 \beta+\gamma_{g_{1}}}{2(1-\alpha)}, \\
& \Delta n_{s_{i}}=\left|\left\langle n_{s_{i}}\right\rangle-N / k\right|=\left|\frac{k \gamma_{g_{1}}-2 N p}{2 k(1-\alpha)}\right|=\frac{N p\left|k / k_{g_{1}}-2\right|}{2 k(1-\alpha)} .
\end{aligned}
$$

with $a \in R$, and $n_{s_{i}}^{\left(t^{\prime}\right)}$ stands for the number of agents choose $s_{i}$ at time $t^{\prime}$. Here, $\alpha \equiv(1-p)(1-m), \beta \equiv N(1-p) m / k$, and $\gamma_{g_{1}} \equiv N p / k_{g_{1}}$. The corresponding equations for $s_{j}$ are also obtained in Supplementary Information.

The mean-field theory is ideally suited for fully connected networks. Indeed, results from the theory and direct simulations agree with each other very well, as shown in Fig. 6. However, in real-world situations, a fully connected topology cannot be expected, and the mean-field treatment will no longer be accurate. For example, we have carried out simulations on square-lattice systems and found noticeable deviations from the mean-field prediction.

To remedy this deficiency, we develop a modified mean-field analysis for MG dynamics on sparsely homogeneous networks (e.g., square lattices or random networks). Due to the limited number of links in a typical large-scale network, it is possible for a failed agent to be surrounded by agents from the same group (who will likewise fail the game). In this case, the failed agent has no minority strategy to imitate (set $\Pi$ is empty) and thus will randomly select one strategy from the $k$ available strategies. From the modified mean-field analysis, which takes this effect into account, we obtain the formulas for $n_{s_{i}}, A_{s_{i}},\left\langle n_{s_{i}}\right\rangle$, and so on. A good agreement with simulation results is shown in Fig. 6 for a square-lattice system.

Stability of strategy grouping states. Our mean-field treatment yields formulas characterizing the stable oscillations associated with the
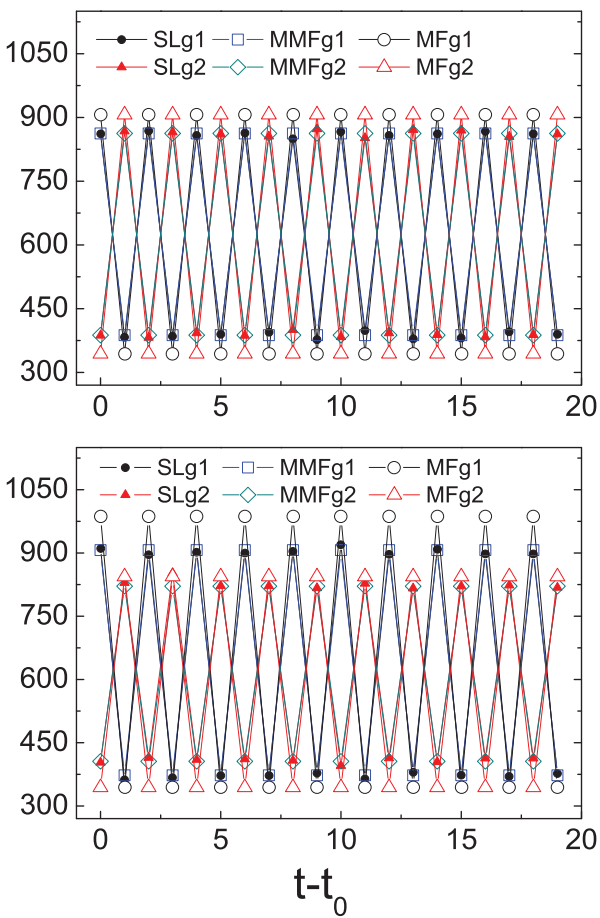

Figure 6 Comparison of the time series of $n_{s_{i}}$ in group $g_{1}$ (and $n_{s j}$ in group $g_{2}$ ) from the mean-field formula [Eq. (6)] and modified mean-field formula [Eq. (15) in Supplementary Information], and from simulations. The upper and lower figures show the results for the grouping state (8; 8) and (7; 9), respectively. The left panels are the results from mean-field theory (MF) and from simulations on fully connected networks (FCN). The right panels are the results from our modified mean-field theory (MMF) and simulations on the square lattice (SL). The system parameters are $N=10000, k=16$, $p=0.45$, and $m=1$. 
grouping state $\left(k_{g_{1}}, k_{g_{2}}\right)$, which include the variance ratio of the pairing groups [Eq. (5)] and time series $n_{s}$ [Eq. (6)].

However, from the simulation result shown in Fig. 3, we see that not all the grouping states are stable in the parameter space. As $p$ is increased, the strategy-grouping state of the smaller $k_{g_{1}}$ becomes stable, and the corresponding branch appears. It is therefore useful to analyze the stability of the grouping state.

In our treatment we have assumed $n_{s_{i}}^{(0)}>N / k$ and $n_{s_{j}}^{(0)}<N / k$. Then, the necessary condition for the grouping state $\left(k_{g_{1}}, k_{g_{2}}\right)$ to become stable is $n_{s_{i}}^{(1)}<N / k$ and $n_{s_{j}}^{(1)}>N / k$. Using the equations of the time series $n_{s}$ for strategy $s_{i}$ and $s_{j}$, [Eq. (6), or see Supplementary Information Eqs. (12) and (13)], we get

$$
\begin{aligned}
& k_{g_{1}}>\frac{k(1-m-p+p m)}{2-m-p+p m} \equiv \xi_{1}(p, m), \\
& k_{g_{2}}<\frac{k}{2-m-p+m p} \equiv \xi_{2}(p, m),
\end{aligned}
$$

where $\xi_{1}$ and $\xi_{2}$ are continuous functions of the parameters $p$ and $m$, and $\xi_{1}+\xi_{2}=k$. The two inequalities in Eq. (8) are nevertheless equivalent to each other. Figure 7 presents a phase diagram in the parameter space, where the curves of $\xi_{1}(p, m)=k^{\prime}\left(k^{\prime}=8,7, \ldots, 1\right)$ are shown. The necessary condition for the strategy-grouping state with $k_{g_{1}}=k^{\prime}$ to be stable is that the parameters $p$ and $m$ are in the upper-right region of the curve $\xi_{1}(p, m)=k^{\prime}$. For certain value of $m$, only when $p>p_{b}\left(m, k^{\prime}\right)$ will the state of $k_{g_{1}}=k^{\prime}$ be stable. While the value of $p_{b}$ from simulation is different from the theoretical value $p_{b}\left(m, k^{\prime}\right)$, our mean-field theory does provide a qualitative explanation for the phenomenon in Fig. 3(a), where more branches of smaller strategy-grouping states become stable as $p$ is increased.

We have also seen in Figs. 2 and 3(a) that, as $p$ approaches 1, the bifurcated branches of different grouping state merge together. For the case of even $k, n_{s}$ fluctuates stably in the grouping state with $k_{g_{1}}=k_{g_{2}}=k / 2$. While, for the case of odd $k, n_{s}$ fluctuates quasi-periodically [see Fig. 1(c)]. In fact, the grouping state always switches between $\left(k_{g}+1, k_{g}\right)$ and $\left(k_{g}, k_{g}+1\right)$, with $k_{g}=(k-1)$ /
2. We can understand the instability and merging of grouping states from Fig. 1(c), and the schematic map in Fig. 5, as follows. As $p$ is increased to $1, \Delta n_{s_{i}}$ and $\Delta n_{s_{j}}$ increase and become comparable to the amplitudes $A_{s_{i}}$ and $A_{s_{j}}$, respectively. Namely, the attendances $n_{s}$ of strategies can be very close to $n_{c}=N / k$. In case where $n_{s}$ of one strategy does not become larger than $n_{c}$ because of noise, i.e., it acts as minority (or majority) strategy twice, then the fluctuation of $n_{s}$ as well as the grouping state are necessarily changed.

Relevance to real-world systems. We have observed the strategygrouping like phenomenon in a subsystem of Shanghai Stock Exchange by analyzing the available time series of daily stock price. In principle, by observing the detailed behavior of the strategies employed by the agents in the market, an unequivocal demonstration of the grouping phenomenon could be made. This, however, is practically impossible. That grouping is a generic phenomenon in real-world systems such as the stock market remains therefore to be speculative. Our confidence, however, comes from the close analog between any subsystem of the stock market and MG system with multiple resources, and the generality of the grouping phenomenon in such systems as established by our analytic theory. It would certainly be interesting to develop approaches to probing directly the grouping phenomenon in realworld complex systems.

Other real-world systems for which our MG model may be applicable include, e.g., hedge-fund portfolios in financial systems, routing issues in computer networks and urban traffic systems, and the selection process of high-school candidates in the college admission system. Specifically, in the Chinese college admission (Gaokao) system, candidates often select their dream colleges according to previous years admission scores, but their destiny actually depends more on the present admission scores determined according to some kind of minority game mechanism. Further, the oscillations of annual admission scores and their trends for a number of colleges, corresponding to the grouping of resources investigated in this paper, is not

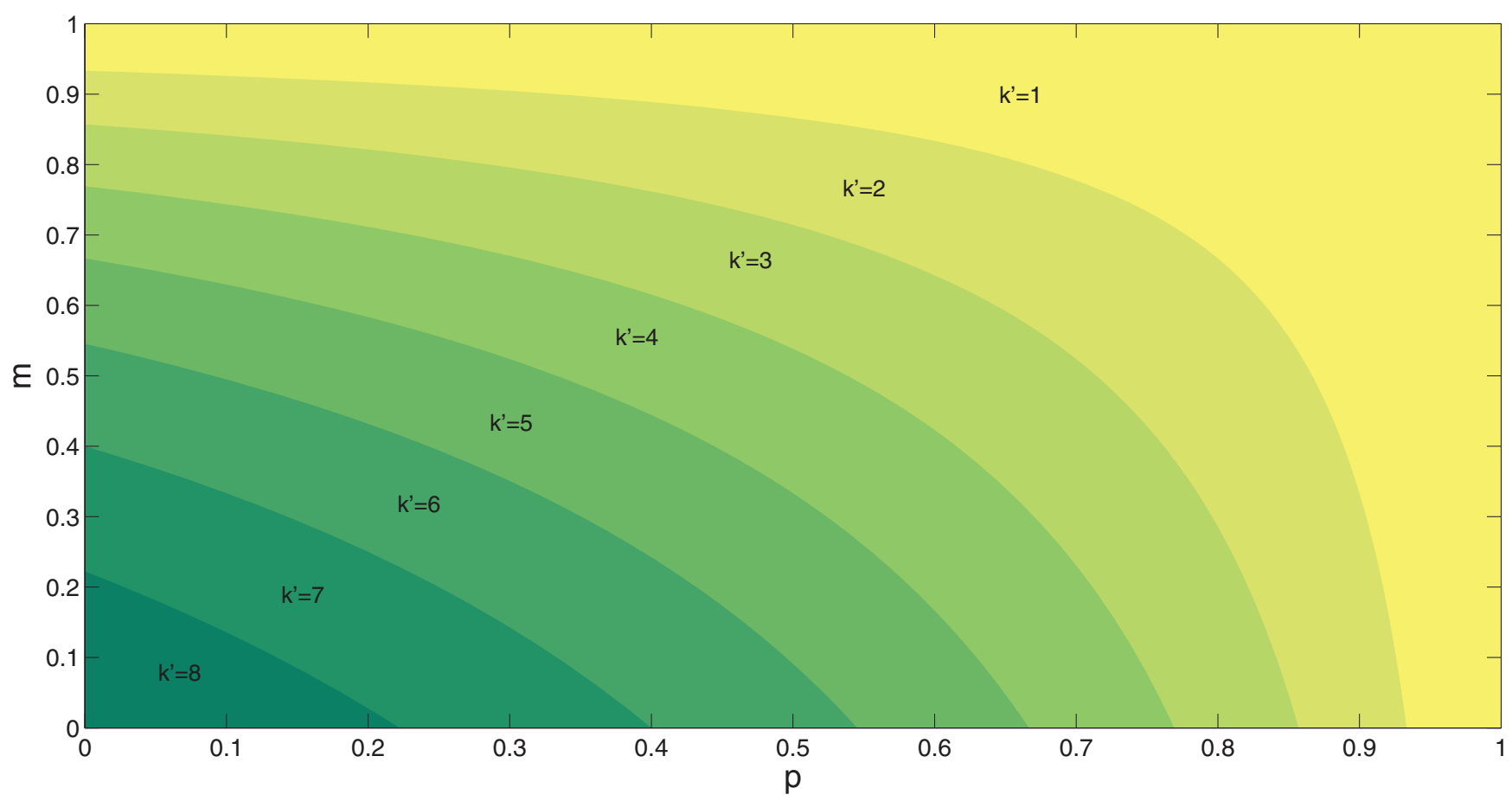

Figure $7 \mid$ Phase diagram indicating the stability region of strategy-grouping states. The stable parameter region of one given grouping state with $k_{g_{1}}=k^{\prime}$ is at the upper right of the corresponding curve of $k^{\prime}$. 

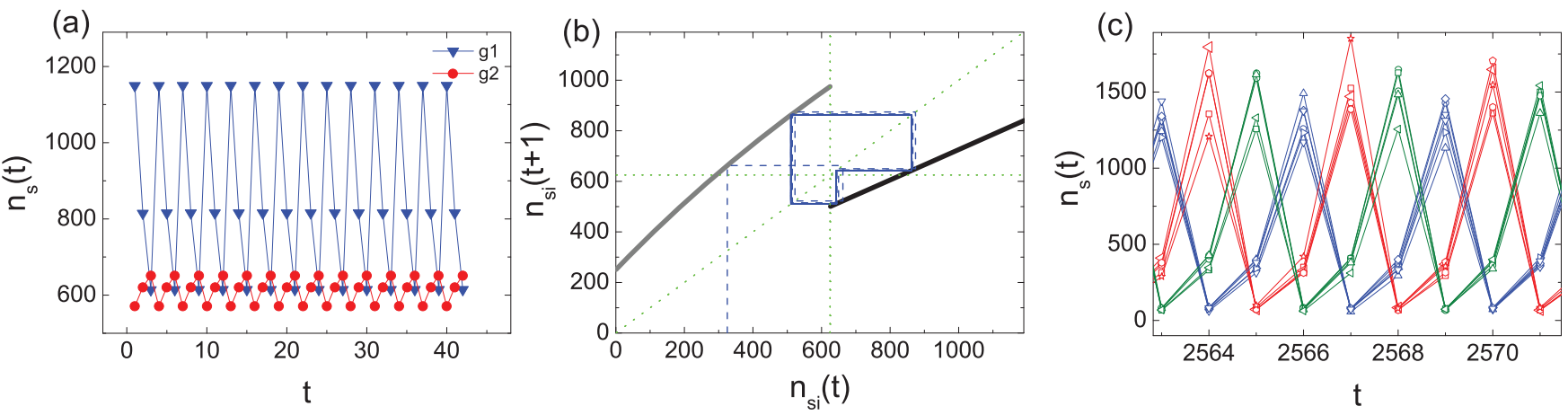

Figure $8 \mid$ (a) Analytical time series $n_{s}(t)$ of period-tripling in the form of 2 groups, and (b) the corresponding mapping from $n_{s_{i}}(t)$ to $n_{s_{i}}(t+1)$ for strategy $s_{i}$ in group $g_{1}$ [see Eq. (14) in Supplementary Information]. The system parameters are $k=16, p=0.25, m=0.2$, and the grouping state $\left(k_{g_{1}}, k_{g_{2}}\right)$ is $(3,13)$. (c) Time series $n_{s}(t)$ of the stable grouping state with period-tripling in the form of 3 groups from simulation on FCN. The system parameters are $k=16, p=0.88, m=1$, and the grouping state $\left(k_{g_{1}}, k_{g_{2}}, k_{g_{3}}\right)$ is $(5,5,6)$.

an uncommon phenomenon in the history of Chinese Gaokao admission system.

\section{Discussion}

Minority game, since its invention about two decades ago, has become a paradigm to study those social and economical phenomena where a large number of agents attempt to make simultaneous decision by choosing one of the available strategies ${ }^{2}$. In the most commonly studied case of single available resource with players' two possible strategies, agents taking the minority strategy are the guaranteed winners. Various minority game dynamics have also received attention from the physics community due to their high relevance to a number of phenomena in statistical physics. It has become more and more common in the modern world that multiple resources are available for various social and economical systems. If the rule still holds that the winning options are minority ones, the questions that naturally arise are what type of collective behaviors can emerge and how they would evolve in the underlying complex system. Our present work aims to address these questions computationally and analytically.

The main contribution and findings of this paper are the following. First, we have constructed a class of spatially extended systems in which any agent interacts with a finite but fixed number of neighbors and can choose either to follow the minority strategy based on information about the neighboring states or to select one randomly from a set of available strategies. The probability to follow the local minority strategy, or the probability of minority preference, is a key parameter determining the dynamics of the underlying complex system. Second, we have carried out extensive numerical simulations and discovered the emergence of a striking collective behavior: as the minority-preference probability is increased through a critical value, the set of available strategies/resources spontaneously break into pairs of groups, where the strategies in the same group are associated with a specific fluctuating behavior of attendance. This phenomenon of strategy grouping is completely self-organized, which we conjecture is the hallmark of MG dynamics with multiple resources. Third, we have developed a mean-field theory to explain and predict the emergence and evolution of the strategy-grouping states, with good agreement with the numerics. Fourth, we have examined a realworld system of a relatively small-scale stock-trading subsystem, and found unequivocal evidence of the grouping phenomenon. Our results suggest grouping of resources as a fundamental type of collective dynamics in multipleresource MG systems.

Finally, we remark that the bifurcation-like phenomena associated with resource grouping in minority-game systems are not limited to the double-grouping (or paired grouping) behavior. In fact, we have also observed phenomena such as period-3 double-grouping [see Fig. 8(a)(b)], and period-3 triplet-grouping bifurcation [see Fig. 8(c)]. Further efforts are warranted to explore various nonlinear dynamical phenomena in minority-game type of systems that describe a large variety of social, economical, and political systems.

1. Brian, W. Arthur, Inductive Reasoning and Bounded Rationality. Ame. Econo. Rev. 84, 406-411 (1994).

2. Gintis, H. Game Theory Evolving (Princeton University Press, Princeton, 2009).

3. Challet, D. \& Zhang, Y.-C. Emergence of Cooperation and Organization in an Evolutionary Game. Physica A 246, 407-418 (1997).

4. Challet, D. \& Marsili, M. Phase Transition and Symmetry Breaking in the Minority Game. Phys. Rev. E 60, 6271(R) (1999).

5. Challet, D., Marsili, M. \& Zecchina, R. Statistical Mechanics of Systems with Heterogeneous Agents: Minority Games. Phys. Rev. Lett. 84, 1824 (2000).

6. De Martino, A., Marsili, M. \& Mulet, R. Adaptive Drivers in a Model of Urban Traffic. Europhys. Lett. 65, 283-289 (2004).

7. Borghesi, C., Marsili, M. \& Miccichè, S. Emergence of Time-horizon Invariant Correlation Structure in Financial Returns by Subtraction of the Market Mode. Phys. Rev. E 76, 026104 (2007).

8. Savit, R., Manuca, R. \& Riolo, R. Adaptive Competition, Market Efficiency, and Phase Transitions. Phys. Rev. Lett. 82, 2203 (1999).

9. Paczuski, M., Bassler, K. E. \& Corral, A. Self-Organized Networks of Competing Boolean Agents. Phys. Rev. Lett. 84, 3185 (2000).

10. Zhou, T., Wang, B.-H., Zhou, P.-L., Yang, C.-X. \& Liu, J. Self-organized Boolean Game on Networks. Phys. Rev. E 72, 046139 (2005).

11. Eguiluz, V. M. \& Zimmermann, M. G. Transmission of Information and Herd Behavior: An Application to Financial Markets. Phys. Rev. Lett. 85, 5659 (2000)

12. Kalinowski, T., Schulz, H.-J. \& Birese, M. Cooperation in the Minority Game with Local Information. Physica A 277, 502-508 (2000).

13. Slanina, F. Harms and Benefits from Social Imitation. Physica A 299, 334-343 (2000).

14. Anghel, M., Toroczkai, Z., Bassler, K. E. \& Korniss, G. Competition-Driven Network Dynamics: Emergence of a Scale-Free Leadership Structure and Collective Efficiency. Phys. Rev. Lett. 92, 058701 (2004).

15. Lo, T. S., Chan, H. Y., Hui, P. M. \& Johnson, N. F. Theory of Networked Minority Game Based on Strategy Pattern Dynamics. Phys. Rev. E 70, 056102 (2004).

16. Lo, T. S., Chan, K. P., Hui, P. M. \& Johnson, N. F. Theory of Enhanced Performance Emerging in a Sparsely Connected Competitive Population. Phys. Rev. E 71, 050101(R) (2005).

17. Johnson, N. F., Hart, M. \& Hui, P. M. Crowd Effects and Volatility in Markets with Competing Agents. Physica A 269, 1-8 (1999).

18. Hart, M., Jefferies, P., Johnson, N. F. \& Hui, P. M. Crowd-Anticrowd Theory of the Minority Game. Physica A 298, 537-544 (2001).

19. Challet, D., De Martino, A. \& Marsili, M. Dynamical Instabilities in a Simple Minority Game with Discounting. J. Stat. Mech. L04004 (2008).

20. Bianconi, G., De Martino, A., Ferreira, F. F. \& Marsili, M. Multi-Asset Minority Games. Quant. Finance 8(3), 225-231 (2008).

21. Xie, Y. B., Wang, B. H., Hu, C.-K. \& Zhou, T. Global Optimization of Minority Game by Intelligent Agents. Eur. Phys. J. B 47, 587-593 (2005).

22. Zhong, L.-X., Zheng, D. F. Zheng, B. \& Hui, P. M. Effects of Contrarians in the Minority Game. Phys. Rev. E 72, 026134 (2005).

23. Moro, E. The Minority Game: an Introductory Guide, in Advances in Condensed Matter and Statistical Physics, ed.by E. Korutcheva and R. Cuerno (Nova Science Publishers, Inc., 2004). 
24. Challet, D., Marsili, M. \& Zhang, Y.-C. Minority Games (Oxford University Press, Oxford, 2005).

25. Yeung, C. H. \& Zhang, Y.-C. Minority Games. Encyclopedia of Complexity and Systems Science 5588-5604 (2009).

\section{Acknowledgement}

ZGH thanks Prof. Ying-Hai Wang, and Matteo Marsili for helpful discussions. This work was partially supported by the NSF of China (Grant Nos. 11275003, 10905026, 11005053 and 11135001). YCL was supported by AFOSR under Grant No. FA9550-10-1-0083.

\section{Author contributions}

ZG Huang, L Huang and YC Lai devised the research project and wrote the paper. JQ Zhang,
ZG Huang and L Huang did the theoretical analysis. JQ Dong and ZG Huang did the numerical simulation and data analysis.

\section{Additional information}

Supplementary information accompanies this paper at http://www.nature.com/ scientificreports

Competing financial interests: The authors declare no competing financial interests.

License: This work is licensed under a Creative Commons

Attribution-NonCommercial-NoDerivative Works 3.0 Unported License. To view a copy of this license, visit http://creativecommons.org/licenses/by-nc-nd/3.0/

How to cite this article: Huang, Z., Zhang, J., Dong, J., Huang, L. \& Lai, Y. Emergence of grouping in multi-resource minority game dynamics. Sci. Rep. 2, 703; DOI:10.1038/ srep00703 (2012) 